TRANSACTIONS OF THE

AMERICAN MATHEMATICAL SOCIETY

Volume 284. Number 2. August 1984

\title{
ON POSITIVE SOLUTIONS OF SOME PAIRS \\ OF DIFFERENTIAL EQUATIONS
}

BY

E. N. DANCER

\begin{abstract}
In this paper, we discuss the existence of solutions, with both components positive, of a Dirichlet problem for a coupled pair of partial differential equations. The main result is proved by using degree theory in cones. We also discuss the asymptotic behaviour of solutions as a parameter tends to zero.
\end{abstract}

In this paper, we discuss the nonnegative solutions of the pair of equations

$$
-u^{\prime \prime}=u(a(1-u)-v), \quad-d v^{\prime \prime}=v(-v+m(u-\gamma))
$$

on $[-L, L], u(-L)=u(L)=v(-L)=v(L)=0$. We assume that $\gamma<1$. This system was discussed in Conway, Gardner and Smoller [6]. These equations arise when one looks for equilibrium solutions of a parabolic system. The system is a model system for a two species predator-prey interaction when both systems undergo diffusion. A more complete discussion of the significance of the equations and the choice of nonlinearities can be found in the introduction to [6].

We prove that there is a $d(L)>0$ such that, if $d \geqslant d(L)$, any nonnegative solution $(u, v)$ of our system satisfies $v(x)=0$ on $[-L, L]$. However, if $d<d(L)$, there is a nonnegative solution $\left(u^{*}, v^{*}\right)$ of (1) such that $u$ and $v$ are both strictly positive on $(-L, L)$. This partially answers a question in [6, p. 316]. In fact, we prove the existence of a connected set of such solutions (parameterized by $a, d$ or $L$ ). We prove this result by using degree theory in cones and by using the calculation of the local index of certain solutions in our earlier work [8]. Our methods seem to be applicable to a great many related problems. We discuss a few of these. In particular, we prove our main results with (1) replaced by a corresponding set of elliptic equations. Finally, we discuss the asymptotic behaviour of positive solutions as $d \rightarrow 0$ and answer another question from [6]. (Once again, we prove our results for the case of elliptic equations.) We also discuss briefly the case where $a(1-u)$ is replaced by $a(1-u)(u-b)$. It turns out that, in certain circumstances, the solution structure in this case can be rather more complicated. We also obtain a few results for some competing systems models.

In $\S 1$, we prove the existence of the solution $(u, v)$ with both components strictly positive on $(-L, L)$. In $\S 2$, we briefly discuss how our method applies in some more general situations. Finally, in $\S 3$, we prove the results on the asymptotic behaviour of solutions as $d \rightarrow 0$.

Received by the editors December 13, 1983.

1980 Mathematics Subject Classification. Primary 34B15, 35J65, 47H15; Secondary 58E07, 92A15.

"1984 American Mathematical Society $(0) 2-9947 / 84 \$ 1 .(0)+\$ .25$ per page 
Throughout, we emphasize the use of positive operator techniques. This has the advantage that it applies to nonselfadjoint problems. It seems natural to use degree theory with respect to cones when studying positive solutions.

I should like to thank the referee for informing me of some additional references.

1. The existence of positive solutions. We consider the more general system

$$
-\Delta u=f(u)-u v, \quad-\Delta v=d^{-1} v(-v+m(u-\gamma))
$$

on a smooth bounded domain $\Omega \subseteq R^{n}$ with Dirichlet boundary conditions. Here $f(y)=a y(1-y)$. (In fact, we could avoid assuming any smoothness on $\Omega$.) The case in the introduction is when $n=1$ and $\Omega=(-L, L)$. As in [6], we assume that $a, d, m, \gamma>0$ and $\gamma<1$. A solution $(u, v)$ is said to be strictly positive if $u(x)>0$ and $v(x)>0$ for $x \in \Omega$.

We first obtain some simple estimates for nonnegative solutions (that is, solutions with $u, v$ both nonnegative in $\Omega$ ), as in [6]. If we look at the point $\bar{x}$ where $u$ has its maximum, we easily see that $a(1-u(\bar{x}))-v(\bar{x}) \geqslant 0$. Since $v(\bar{x}) \geqslant 0$, it follows that $u(x) \leqslant 1$ in $\Omega$. Similarly, by considering the point $x_{1}$ where $v$ has its maximum, we see that $v\left(x_{1}\right) \leqslant m\left(u\left(x_{1}\right)-\gamma\right) \leqslant m(1-\gamma)$. Moreover, since $u$ is a solution of a linear equation and since $u$ is nonnegative, we see that $u(x)=0$ on all of $\Omega$ or $u(x)>0$ on all of $\Omega$. A similar property holds for $v$. Moreover, there can be no nontrivial nonnegative solution $(u, v)$ such that $u(x)=0$ on $\Omega$. This follows, since otherwise, as before $v\left(x_{1}\right) \leqslant m\left(u\left(x_{1}\right)-\gamma\right)=-m \gamma<0$. (Here $x_{1}$ is the point where $v$ has its maximum.) This contradicts our assumption that $v$ is nonnegative.

We need the following well-known lemma for the equation

$$
-\Delta u=f(u) \text { in } \Omega, \quad u=0 \quad \text { on } \partial \Omega .
$$

Note that our system reduces to this if we look for solutions with $v=0$.

LEMMA 1. (i) If $a \leqslant \lambda_{1}$, (3) has no nontrivial nonnegative solution.

(ii) If $a>\lambda_{1}$, there is a unique nonnegative nontrivial solution $u_{a}$ of (3).

(iii) If $\tilde{u} \in C(\bar{\Omega}) \cap \dot{W}^{1,2}(\Omega)$, if $0 \leqslant \tilde{u}(x) \leqslant 1$ in $\Omega$, if $\left.\tilde{u}\right|_{\partial \Omega} \leqslant 0$ and if

$$
-\Delta \tilde{u}(x) \leqslant a \tilde{u}(x)(1-\tilde{u}(x)) \text { in } \Omega
$$

in the sense of distributions, then there is a solution $u$ of (3) such that $u \geqslant \tilde{u}$.

(iv) For each compact subset $K$ of $\Omega$ and for each $\alpha$ in $(0,1)$, there is an $\tilde{a}>0$ such that $u_{a}(x) \geqslant \alpha$ on $K$ if $a \geqslant \tilde{a}$.

Proof. Parts (i)-(iii) are classical results. For example, (i) and (ii) could be proved by the classical techniques in Pimbley [14] (see, for example, Berestycki [4]). This could also be proved by positive operator theory as in Amann [2]. The key point is that $y f^{\prime}(y)<f(y)$ on $(0,1)$. Part (iii) can also be found in [2]. (Note that $u(x)=1$ is a supersolution.) To prove (iv), it suffices to construct a suitable subsolution $\tilde{u}$. To do this, we choose a function $s$ such that $s$ is smooth in $\Omega, s=h_{1}$ near $\partial \Omega, s(x)=\alpha$ in a neighbourhood of $K$, and $0<s(x) \leqslant \alpha$ in $\Omega$. Here $h_{1}$ is the first eigenfunction of $-\Delta$ on $\Omega$ with Dirichlet boundary conditions. Since $\Delta s$ is then bounded on $\Omega$, it is easy to see that $s$ is a subsolution if $a$ is sufficiently large. (Note that $1-s(x) \geqslant 1-\alpha$ on $\Omega$.) Thus (iv) follows from (iii). 
The operator $L_{a}=\left(-\Delta+\gamma m d^{-1} I\right)^{-1} d^{-1} m u_{a} I$ is a compact operator on $C(\bar{\Omega})$. Here, and henceforth when we write $(-\Delta+\alpha I)^{-1}$, we assume Dirichlet boundary conditions. We use $r$ to denote the spectral radius.

If $a \leqslant \lambda_{1}$, we define $u_{a}=0$. Thus, in this case, $r\left(L_{a}\right)=0$. We now state our main result.

THEOREM 1. (i) If $r\left(L_{a}\right) \leqslant 1$, the only nonnegative solutions $(u, v)$ of (2) have $v(x)=0$ on $\Omega$.

(ii) If $r\left(L_{a}\right)>1$, there is a strictly positive solution $\left(u^{*}, v^{*}\right)$ of $(2)$.

Remark. As we will see in Proposition 2, there is at most one $a$ for which $r\left(L_{a}\right)=1$. Moreover, if $r\left(L_{a^{*}}\right)=1$, then $r\left(L_{a}\right)<1$ for $a<a^{*}$ and $r\left(L_{a}\right)>1$ for $a>a^{*}$. We will obtain alternative conditions equivalent to $r\left(L_{a}\right) \leqslant 1$ or $>1$ in Proposition 2.

Before proving Theorem 1, we need some preliminaries. First note that we can calculate $r\left(L_{a}\right)$ in the subspace $C_{0}(\bar{\Omega}) \equiv\left\{u \in C(\bar{\Omega}):\left.u\right|_{\partial \Omega}=0\right\}$ since $L_{a}$ maps $C(\bar{\Omega})$ into $C_{0}(\bar{\Omega})$. Now the set $K$ of nonnegative functions in $C_{0}(\bar{\Omega})$ is a closed cone in $C_{0}(\bar{\Omega})$ in the sense of Krasnosel'skii [12, p. 17] and $L_{a}(K) \subseteq K$. (In the usual terminology, $L_{a}$ is positive.) By the maximum principle, $L_{a}$ maps nonnegative elements of $K$ to elements of $C_{0}(\bar{\Omega})$ which are positive in $\Omega$. With the terminology of [8], $L_{a}$ maps $K \backslash\{0\}$ to demi-interior elements of $K$ and so $L_{a}$ is a demi-interior operator. Here an element $x$ in a cone $C$ in a Banach space $E$ is said to be demi-interior to $C$ if $l(x)>0$ for every $l \in C^{*} \backslash\{0\}$, where $C^{*}=\left\{l \in E^{*}: l(x) \geqslant 0\right.$ on $C$ \}. Moreover, a positive operator $L$ is demi-interior if, for each $x \in C \backslash\{0\}$, $L^{n} x$ is demi-interior to $C$ for some nonnegative integer $n$. Implicit in the above was the following simple lemma.

Lemma 2. Suppose $x \in K \subseteq C_{0}(\bar{\Omega})$. Then $x$ is demi-interior to $K$ if and only if $x(t)>0$ on all of $\Omega$.

Proof. The necessity of the condition $x(t)>0$ on $\Omega$ is obvious by using Dirac measures. To prove sufficiency, suppose $l \in K^{*}$. It follows easily from Schaefer [17, Theorem 5.5.4] that $l$ extends to a positive linear functional on $C(\bar{\Omega})$. Thus, by the Riesz representation theorem (cf. Rudin [16, p. 40]), $l$ is given by integration with respect to a positive Borel measure $\mu$. ( $\mu$ is not unique as we could change it by measures supported in $\partial \Omega$.) Hence $l(x)=\int_{\bar{\Omega}} x d \mu$. Since $\mu$ is positive and $x(t)>0$ in $\Omega$, it follows that, if $l(x)=0$, then the support of $\mu$ is contained in $\partial \Omega$. However, this implies that $l$ vanishes on $C_{0}(\bar{\Omega})$ and so the result follows.

Now it is a well-known result that, when $r\left(L_{a}\right)>0$ (that is, when $\left.a>\lambda_{1}\right)$, there is an $h_{a} \in K \backslash\{0\}$ such that $L_{a} h_{a}=r\left(L_{a}\right) h_{a}$ and that $h_{a}$ is the only positive eigenvector of $L_{a}$. (For example, this follows since $L_{a}$ is a demi-irreducible operator. However, it is a classical result.) We consider the operators

$$
L_{a, t}=\left(-\Delta+\left(m \gamma d^{-1}+t\right) I\right)^{-1}\left(d^{-1} m u_{a}+t\right) I \text { for } t \geqslant 0 .
$$

By arguments similar to those above, $L_{a, t}$ has similar spectral properties to $L_{a}$. Moreover, if $L_{a} h_{a}=h_{a}$, we see by simple calculation that $L_{a, t} h_{a}=h_{a}$ and conversely. Thus $r\left(L_{a}\right)=1$ if and only if $r\left(L_{a, t}\right)=1$ for some (and hence all) $t \geqslant 0$. By 
continuous dependence of the spectral radius of compact operators (cf. Kato [11, Theorem 4.3.1 and \$4.3.5]), it follows that, if $r\left(L_{a}\right)<1$, then $r\left(L_{a, t}\right)<1$ for all $t \geqslant 0$, while if $r\left(L_{a}\right)>1$, then $r\left(L_{a, t}\right)>1$ for all $t \geqslant 0$. We say $u<v$ if $v-u \in$ $K \backslash\{0\}$.

Proof of Theorem 1(i). If $(u, v)$ is a nonnegative solution of $(2)$ with $v \neq 0$, then

$$
-\Delta u=a u(1-u)-u v \leqslant a u(1-u) .
$$

Thus, by Lemma 1(iii), $u_{a} \geqslant u$. Now

$$
-\Delta v+m \gamma d^{-1} v=d^{-1} v(-v+m u)<d^{-1} m u_{a} v .
$$

Hence $v<\left(-\Delta+\gamma m d^{-1} I\right)^{-1} d^{-1} m u_{a} v$, that is, $v<L_{a} v$. By a standard result (cf. [12, Theorem 2.5]), $r\left(L_{a}\right) \geqslant 1$. It remains to obtain a contradiction if $r\left(L_{a}\right)=1$. If $r\left(L_{a}\right)=1$, then, by the Krein-Rutman theorem (cf. [17, p. 265]) there is an $\tilde{f} \in K^{*} \backslash\{0\}$ such that $\tilde{f}=L_{a}^{*} \tilde{f}$. Thus $\tilde{f}(x)=\left(L_{a}^{*} \tilde{f}\right)(x)=\tilde{f}\left(L_{a} x\right)>0$ if $x \neq 0$ (since $L_{a} x$ is demi-interior to $\left.K\right)$. Hence $\tilde{f}(x)>0$ if $x \in K \backslash\{0\}$. Since $L_{a} v-v<0$, it follows that $\tilde{f}\left(L_{a} v\right)-\tilde{f}(v)<0$. This is impossible since $\tilde{f}(v)=\left(L_{a}^{*} \tilde{f}\right)(v)=\tilde{f}\left(L_{a} v\right)$. This completes the proof. (The same argument as in the last part of the proof could be used to prove a general result for demi-interior operators.)

Before proving the second part of Theorem 1, we need one more lemma. If $C$ is a cone in a Banach space $E$, and $z \in C \backslash\{0\}$, let $z^{\perp}=\left\{l \in C^{*}: l(z)=0\right\}, \bar{S}_{z}=\{x \in$ $E: l(x)=0$ for all $l$ in $\left.z^{\perp}\right\}$ and $\bar{C}_{z}=\left\{x \in E: l(x) \geqslant 0\right.$ for all $l$ in $\left.z^{\perp}\right\}$.

LEMMA 3. If $E=C_{0}(\bar{\Omega}) \oplus C_{0}(\bar{\Omega})$, if $C=K \oplus K$ and if $z=(u, 0)$ where $u(x)>0$ in $\Omega$, then $\bar{S}_{z}=C_{0}(\bar{\Omega}) \oplus\{0\}$ and $\bar{C}_{z}=C_{0}(\bar{\Omega}) \oplus K$.

Proof. Note that $l \in E^{*}$ if and only if $l=\left(f_{1}, f_{2}\right)$ where $f_{i} \in C_{0}(\bar{\Omega})^{*}$. Moreover, $l$ is positive if and only if $f_{1}$ and $f_{2}$ are positive. Now $l=\left(f_{1}, f_{2}\right) \in z^{\perp}$ if and only if $f_{1}(u)=0$. As in the proof of Lemma 2, this is only possible if $f_{1}=0$. Thus $z^{\perp}=\{0\} \oplus K^{*}$. Hence $(x, y) \in S_{z}$ if and only if $l(y)=0$ for all $l$ in $K^{*}$ and thus if and only if $y=0$. Similarly $(x, y) \in \bar{C}_{z}$ if and only if $l(y) \geqslant 0$ for all $l \in K^{*}$, that is, $y \in K$. Hence the result follows.

Proof of Theorem 1(ii). We choose $p>m \gamma d^{-1}$ such that $a(1-u)-v+p \geqslant 0$ and $p-v+m(u-\gamma) \geqslant 0$ if $0 \leqslant u \leqslant 2$ and $v \leqslant m(2-\gamma)$. We define $A_{a}: T \rightarrow C$ by

$$
A_{a}(u, v)=(-\Delta+p I)^{-1}\left(u[a(1-u)-v+p], d^{-1} v[d p-v+m(u-\gamma)]\right),
$$

where $T=\{(u, v) \in C: u(x) \leqslant 2, v(x) \leqslant m(2-\gamma)$ on $\Omega\}$. Here we are abusing notation slightly by using $(-\Delta+p I)^{-1}$ to also denote the obvious extension of this operator to ordered pairs. Note that the fixed points of $A$ are the solutions of (2). It is easy to see that $A$ is completely continuous and Fréchet differentiable. We will use degrees relative to the cone $C$. Since any fixed point of $A$ is a solution of (2) and thus satisfies $u(x) \leqslant 1$ and $v(x) \leqslant m(1-\gamma)$ on $\Omega$ (by our earlier estimates), it follows that all solutions lie in the interior of $T$ (where the interior is with respect to $C$ ). Thus, by homotopy invariance $\operatorname{deg}_{C}\left(I-A_{a}\right.$, int $\left.T\right)$ is independent of $a$. If $a<\lambda_{1}$, then by 
our earlier results, the only solution is $(0,0)$. By Theorem 1 in [8], this solution will have index 1 if $r\left(A_{a}^{\prime}(0,0)\right)<1$. Hence it will follow that

$$
\operatorname{deg}_{C}\left(I-A_{a}, \text { int } T\right)=1
$$

for all $a$ if we prove that $r\left(A_{a}^{\prime}(0,0)\right)<1$ for $a<\lambda_{1}$. Now by an easy calculation

$$
A_{a}^{\prime}(0,0)(u, v)=\left((-\Delta+p I)^{-1}(p+a) u,(-\Delta+p I)^{-1}\left(p-m d^{-1} \gamma\right) v\right) .
$$

Thus it suffices to prove that $(-\Delta+p I)^{-1}(p+a) I$ and $(-\Delta+p I)^{-1}\left(p-m d^{-1} \gamma\right) I$ each has spectral radius less than 1 . These results follow easily. For example, by the spectral mapping theorem the first of these operators has spectra

$$
\left\{(\lambda+p)^{-1}(p+a): \lambda \text { is an eigenvalue of }-\Delta \text { with Dirichlet boundary conditions }\right\}
$$

and thus all its spectra has modulus less than 1 if $a<\lambda_{1}$. (For future reference, note that the same argument shows that $r\left(A_{a}^{\prime}(0,0)\right)>1$ if $a>\lambda_{1}$.)

Secondly, we prove that, if $a>\lambda_{1}$, then $(0,0)$ is an isolated solution with index 0 . It suffices to prove that $h \neq A_{a}^{\prime}(0,0) h$ if $h \in C \backslash\{0\}$ and if $a>\lambda_{1}$ because we proved above that $r\left(A_{a}^{\prime}(0,0)\right)>1$ if $a>\lambda_{1}$ and it is easy to show that $\bar{S}_{0}=E$. (We use Theorem 1 in [8] again.) If $h=(r, k) \in C \backslash\{0\}$ and $h=A_{a}^{\prime}(0) h$, then $r=$ $(-\Delta+p I)^{-1}(p+a) r$ and $k=(-\Delta+p I)^{-1}\left(p+m d^{-1} \gamma\right) k$. Now, by arguments similar to those in the previous paragraph, both of these operators have spectral radius not equal to 1 . Hence, since $r, k \in K$, the required result follows from our earlier comments on the eigenvectors of these two operators.

Thirdly, we prove that, if $r\left(L_{a}\right)>1$, then the solution $z=\left(u_{a}, 0\right)$ is also isolated and has index zero. A simple calculation shows that

$$
A^{\prime}(z)(r, k)=(-\Delta+p I)^{-1}\left(\left[a\left(1-2 u_{a}\right)+p\right] r-u_{a} k, d^{-1}\left[m\left(u_{a}-\gamma\right)+p\right] k\right) .
$$

To prove $z$ is isolated, we need only prove that $h \neq A^{\prime}(z) h$ if $h \in \bar{W}_{z} \backslash\{0\}$ (cf. [8, $\S 2])$. Now, if $(r, k)$ is an eigenvector of $A^{\prime}(z)$ corresponding to $\lambda=1$, then

$$
-\Delta r=a\left(1-2 u_{a}\right) r \text { or } k=L_{a} k
$$

(plus the boundary condition for $r$ ) has a nontrivial solution. Note that $(r, k) \in \bar{W}_{z}$ implies that $k \in K$ (by Lemma 3). Since $r\left(L_{a}\right)>1$, our earlier comments imply that the equation $k=L_{a} k$ has no nontrivial solution in $K$. Thus, if $(r, k) \in \bar{W}_{z} \backslash\{0\}$ and if $(r, k)=A^{\prime}(z)(r, k)$, then $r$ must be a nontrivial solution of

$$
-\Delta r=a\left(1-2 u_{a}\right) r \text { in } \Omega, \quad r=0 \quad \text { on } \partial \Omega \text {. }
$$

However, the proof of Lemma 1 (cf. [4 or 14]) shows that this does not occur and hence our solution is isolated. By Lemma $3, S_{z}=C_{0}(\bar{\Omega}) \oplus\{0\}$. Now $M=\{0\} \oplus$ $C_{0}(\bar{\Omega})$ is a complement to $S_{z}$ in $E$. Thus, by Theorem 1 in [8] and by Remark 3 after Lemma 3 of [8], $z$ will have index zero provided that $r\left(\left.P A^{\prime}(z)\right|_{M}\right)>1$, where $P$ is the projection onto $M$ parallel to $S_{z}$. (In other words, $P(x, y)=(0, y)$.) Thus $z$ has index zero if $r\left(L_{a, t}\right)>1$ (for $t=p-m d^{-1} \gamma$ ). As before, we see that our assumption that $r\left(L_{a}\right)>1$ implies that $r\left(L_{a, t}\right)>1$ for all $t \geqslant 0$, and hence we have proved the required result. 
We now complete the proof of Theorem 1(ii). Suppose that the result is false. Then, by our earlier comments, the only nonnegative solutions of our equation are $(0,0)$ and $\left(u_{a}, 0\right)$. Thus

$$
\operatorname{deg}_{C}\left(I-A_{a}, T\right)=\operatorname{index}_{C}\left(I-A_{a},(0,0)\right)+\operatorname{index}_{C}\left(I-A_{a},\left(u_{a}, 0\right)\right) .
$$

However, this is impossible by what we have proved since the left-hand side is 1 while the right-hand side is $0+0=0$.

By using standard arguments (cf. Dancer [7] or Rabinowitz [15]), one could show that, if $r\left(L_{a}\right)>1$ for some $a$, then there is an unbounded connected subset $T$ of solutions in $C \times\left[a^{*}, \infty\right)$ such that $(u, v)$ is a strictly positive solution if $(u, v, a) \in$ $T \backslash\left\{\left(u_{a^{*}}, 0, a^{*}\right)\right\}$ and such that $\left(u_{a^{*}}, 0, a^{*}\right) \in T$. (The important point is that the strictly positive solutions have nonzero index sum in $C$.) Note that, as before, $r\left(L_{a^{*}}\right)=1$.

We now want to return to discuss the condition on $r\left(L_{a}\right)$. Our main results on $r\left(L_{a}\right)$ are summarized in the following

Proposition 1. (i) $r\left(L_{a}\right) \geqslant 1$ implies that $a>\lambda_{1}$.

(ii) $r\left(L_{a}\right)$ is strictly increasing in a if $a>\lambda_{1}$.

(iii) There is at most one $a^{*}$ such that $r\left(L_{a^{*}}\right)=1$.

(iv) There is an $a^{*}$ such that $r\left(L_{a^{*}}\right)=1$ if and only if $(1-\gamma) m>\lambda_{1} d$.

(v) Let $c$ be defined by the property that $\left\|u_{c}\right\|_{\infty}=\gamma$, where \|\|$_{\infty}$ denotes the supremum norm. Then $a^{*} \geqslant c$ and $a^{*} \rightarrow c$ as $d \rightarrow 0$ (where the other parameters are kept fixed).

(vi) $r\left(L_{a}\right)<1$ if and only if the first eigenvalue of $-d \Delta-m\left(u_{a}-\gamma\right) I$ (with Dirichlet boundary conditions) is positive.

Proof. (i) $L_{a}=0$ if $a \leqslant \lambda_{1}$ since $u_{a}=0$ in this case. Thus $r\left(L_{a}\right) \geqslant 1$ implies that $a>\lambda_{1}$.

(ii) To prove this, we first show that $u_{b}(x) \geqslant u_{a}(x)$ on $\Omega$ if $b>a>\lambda_{1}$. Now, we easily see that $u_{a}$ is a subsolution of (4) (with $a$ replaced by $b$ ). Since $0<u_{a}(x) \leqslant 1$ in $\Omega$, there is a subset of $\Omega$ of positive measure such that the inequality in the definition of subsolution is strict. It follows easily that the solution $\tilde{u}_{1}$ of $-\Delta u+2 b u$ $=b u_{a}\left(3-u_{a}\right)$ satisfies $\tilde{u}_{1}(x)>u_{a}(x)$ in $\Omega$. (Since if $g(x) \geqslant 0$ on $\Omega$ and if $g$ is positive on a set of positive measure, then the solution of

$$
-\Delta z+2 b z=g \quad \text { in } \Omega, \quad z=0 \quad \text { on } \partial \Omega
$$

is strictly positive in $\Omega$.) Since by $(3-y)$ is increasing on $[0,1]$ and since $u(x)=1$ is a supersolution, then a well-known result (cf. Amann [2]) ensures that $\tilde{u}_{1}(x)$ is the first term of an increasing sequence of functions which converge to $u_{b}$. Thus $u_{b}(x) \geqslant$ $\tilde{u}_{1}(x)>u_{a}(x)$ in $\Omega$, as required. (It is possible to give a more abstract proof of this by showing that the map $a \rightarrow u_{a}$ is differentiable and by using comparison results for positive linear operators to prove $r\left(G^{\prime}\left(u_{a}\right)\right)<1$, where $G(u)=$ $(-\Delta+p I)^{-1}(a u(1-u)+p u)$ for $u \in C_{0}(\bar{\Omega})$, and $p>2 a$.) It follows easily that $L_{b}(x)>L_{a}(x)$ if $x>0$ and $b>a>\lambda_{1}$. Suppose $\tilde{f} \in K^{*} \backslash\{0\}$ is an eigenfunction corresponding to $r\left(L_{a}\right)$. (The Krein-Rutman theorem ensures that $\tilde{f}$ exists since it is easy to prove that $r\left(L_{a}\right)>0$.) Since $L_{a}$ maps $K \backslash\{0\}$ to demi-interior points, it 
follows easily that $\tilde{f}(y)>0$ for $y \in K \backslash\{0\}$. Suppose by way of contradiction that $b>a$ and $r\left(L_{b}\right)=r\left(L_{a}\right)$. Then there exists $x_{b} \in K \backslash\{0\}$ such that $r\left(L_{b}\right) x_{b}=L_{b} x_{b}$. Then $r\left(L_{a}\right) x_{b}=r\left(L_{b}\right) x_{b}=L_{b}\left(x_{b}\right)>L_{a} x_{b}$.

Thus

$$
\begin{aligned}
r\left(L_{a}\right) \tilde{f}\left(x_{b}\right) & >\tilde{f}\left(L_{a} x_{b}\right) \quad(\text { since } \tilde{f} \text { is strictly positive }) \\
& =L_{a}^{*} \tilde{f}\left(x_{b}\right)=r\left(L_{a}\right) \tilde{f}\left(x_{b}\right) .
\end{aligned}
$$

Hence we have a contradiction and thus $r\left(L_{b}\right)>r\left(L_{a}\right)$ if $b>a>\lambda_{1}$.

(iii) follows trivially from (ii).

(iv) By Lemma $1, u_{a} \rightarrow 1$ in $L^{p}(\Omega)$ as $a \rightarrow \infty$ for all $p<\infty$. It follows easily by considering $L_{a}$ as an operator on $L^{p}(\Omega)$ and by using the continuity of the spectral radius that $r\left(L_{a}\right) \rightarrow r(F)$ as $a \rightarrow \infty$, where $F=\left(-\Delta+\gamma m d^{-1} I\right) d^{-1} m I$. (Alternatively one could work on $C_{0}(\bar{\Omega})$ and use collective compactness as in [3].) Since $r\left(L_{a}\right)=0$ if $a \leqslant \lambda_{1}$, we see that $a^{*}$ exists if and only if $r(F)>1$. Since the spectrum of $F$ is $\left\{\left(\lambda+\gamma m d^{-1}\right) d^{-1} m: \lambda \in \sigma(-\Delta)\right\}, r(F)>1$ if and only if

$$
d^{-1} m\left(\lambda_{1}+\gamma d^{-1} m\right)^{-1}>1
$$

that is if and only if $(1-\gamma) d^{-1} m>\lambda_{1}$. (Note for future reference that this always holds if $d$ is sufficiently small and the other parameters are kept fixed.) Thus $a^{*}$ exists if and only if $(1-\gamma) m>\lambda_{1} d$.

(v) If $a \leqslant c,\left\|u_{a}\right\|_{\infty} \leqslant \gamma$, and thus $L_{a} \leqslant\left(-\Delta+m \gamma d^{-1}\right) d^{-1} m \gamma I$ (in the positive operator sense). Since the second operator is easily seen to have spectral radius less than 1 , it follows from [12, p. 94] that $r\left(L_{a}\right)<1$ as well. Thus $a^{*}>c$. To see that $a^{*} \rightarrow c$ as $d \rightarrow 0$ (and the other parameters are held fixed), note that if $z>c$, then $\left\|u_{z}\right\|_{\infty}>\gamma$. It follows easily that there is an open subset $D$ of $\Omega$ with smooth boundary such that $u_{z}(x)>\gamma_{1}>\gamma$ on $\bar{D}$. Hence, if $b>z$, then $u_{b}(x)>\gamma_{1}$ on $\bar{D}$. Let $\tilde{\lambda}_{1}$ denote the first eigenvalue of $-\Delta$ with Dirichlet boundary conditions on $D$ and let $\tilde{h}_{1}$ denote the corresponding first eigenfunction. We define $w(x)$ to be $\tilde{h}_{1}(x)$ on $D$ and to be zero otherwise. Now $d^{-1} \gamma m\left(u_{b}(x)-\gamma\right) \geqslant 2 \tilde{\lambda}_{1}$ on $\bar{D}$ if $d$ is small and $b \geqslant z$. It follows easily that, if $d$ is small and $b \geqslant z$,

$$
-\Delta w \leqslant d^{-1} \gamma m w\left(u_{b}-\gamma\right)
$$

on $\Omega$ in the sense of distributions (cf. [13]). Thus we see that $L_{b} w \geqslant w$ if $d$ is small and if $b \geqslant z$. By Theorem 2.4 in [12], it follows that $r\left(L_{b}\right) \geqslant 1$ if $d$ is small and $b \geqslant z$. Thus $a^{*} \leqslant z$ if $d$ is small and the result follows.

(vi) As is well known, the smallest eigenvalue $\tilde{\lambda}$ of $-d \Delta-m\left(u_{a}-\gamma\right)$ has a corresponding eigenfunction $\tilde{h}$ which is positive on $\Omega$. Now, by a simple calculation, $\left(-\Delta+\gamma m d^{-1} I\right) \tilde{h}>d^{-1} m u_{a} \tilde{h}$ if $\tilde{\lambda}_{1}$ is positive. (Recall that $u>v$ means that $u \geqslant v$ and $u \neq v$.) By applying $\left(-\Delta+\gamma m d^{-1} I\right)^{-1}$, we find that $L_{a} \tilde{h}<\tilde{h}$. If $\tilde{f}$ is defined as in the proof of (ii), we see that $\tilde{f}\left(L_{a} \tilde{h}\right)<\tilde{f}(\tilde{h})$. (Remember $f(u)>0$ if $u>0$.) As in (ii), it follows that $r\left(L_{a}\right) \tilde{f}(\tilde{h})<\tilde{f}(\tilde{h})$. Thus, since $\tilde{f}(\tilde{h})>0, r\left(L_{a}\right)<1$. Similarly, if $\tilde{\lambda}$ is negative, we find that $L_{a} \tilde{h}>\tilde{h}$ and thus $r\left(L_{a}\right)>1$. If $\tilde{\lambda}=0, L_{a} \tilde{h}=\tilde{h}$ and thus $r\left(L_{a}\right)=1$. This completes the proof of Proposition 1 . 
Suppose $a>\lambda_{1}$. It follows from (vi) and an easy calculation that $r\left(L_{a}\right)<1(>1)$ implies that $\left(u_{a}, 0\right)$ is stable (unstable) for the corresponding parabolic equation (as in [6]). Here stability means linearized stability (though in suitable spaces, this is equivalent to stability in the usual sense).

We could use alternative parameterizations. In [6], $d$ was used as a parameter and not much needs to be changed. One difference is that it is easy to check that there is always a positive $d$ for which $r\left(L_{a}\right)=1$. (One uses similar arguments to above to show that $r\left(L_{a}\right)>1$ if $d$ is small and $r\left(L_{a}\right)<1$ if $d$ is large.) The only point that needs checking is the uniqueness of $d$ for which $r\left(L_{a}\right)=1$. To prove this, one can use the variational characterization of eigenvalues or use a theorem of Hess and Kato [10]. (The second method is also valid for nonselfadjoint problems.) We could also parametrize by $\Omega$. More precisely, we could replace $\Omega$ by $L \Omega$ and use $L$ as a parameter, though it is unclear that there is unique $L$ such that $r\left(L_{a}\right)=1$ unless we assume that $L_{1} \Omega \subseteq L_{2} \Omega$ if $0<L_{1} \leqslant L_{2}$. (This holds if $\Omega$ is convex.) Once again, with both $d$ and $L$ parametrizations, one can show the existence of connected sets of solutions.

It seems likely that the strictly positive solution is unique when it exists. Any proof of this is likely to show that the solution is an attractor, as conjectured in [6]. Note that the uniqueness question seems to be related to uniqueness problems for decreasing maps because the pair of equations can be reduced to a single equation involving a decreasing mapping (by solving the first equation for $u$ as a function of $v$ ). In a later paper, we will prove the uniqueness of the strictly positive solution if $n=1$ and $d$ is sufficiently small (and positive).

2. Generalizations. The method we used in $\$ 1$ seems to be a very flexible method for proving the existence of solutions. In this section, we consider rather briefly some generalizations to illustrate this.

Firstly, the results of $\S 1$ still hold if we replace $\Delta$ by a general second order elliptic operator (not necessarily selfadjoint and different operators in the two equations) and for other boundary conditions. Note that, if $\Omega$ is not reasonably regular, it is necessary to work in the space $L^{\infty}(\Omega)$ or $L^{p}(\Omega)$ for large $p$. Moreover, our proof of the existence of the strictly positive solution $\left(u^{*}, v^{*}\right)$ does not use the specific nonlinearity a great deal except on the subspace where $v=0$. (Even this ca be weakened as we see later.) Our proof of the existence of a solution $\left(u^{*}, v^{*}\right)$ for $d$ sufficiently small can also be used if we take $f(y)$ to be $a y(y-b)(1-y)$ instead of ay $(1-y)$, where $0<b<\frac{1}{2}$, at least when $n=1$ and $\Omega=(-L, L)$. (If $b \geqslant \frac{1}{2}$, it can be shown that there is no nontrivial nonnegative solution. This is still true if $n>1$ provided that $\Omega$ is convex.) We assume that we are in the case where (3) has 2 positive solutions $u_{1}, u_{2}$. (When this occurs is discussed in [6].) It is not difficult to show that $u_{1}<u_{2}$. Moreover, it can be shown (cf. [18]) that, under the above assumptions, $-h^{\prime \prime}-f^{\prime}\left(u_{i}\right) h$ with Dirichlet boundary conditions is invertible for $i=1,2$. Assume now that $\left\|u_{2}\right\|_{\infty}>\gamma>\left\|u_{1}\right\|_{\infty}$, where \|\|$_{\infty}$ denotes the sup norm. By similar arguments to those in $\S 1$, one finds that there is a $d_{2}>0$ such that the system of equations has no strictly positive solution if $d \geqslant d_{2}$ while a strictly positive 
solution exists if $d<d_{2}$. Here $d_{2}$ is the unique positive value of $d$ for which $-d h^{\prime \prime}=m\left(u_{2}-\gamma\right) h$ has a positive solution (satisfying the boundary conditions). The idea in the proof is to show that $(0,0)$ has index 1 in $C,\left(u_{1}, 0\right)$ has index -1 always, while $\left(u_{2}, 0\right)$ has index 0 if $d<d_{2}$. Note that, as before, the sum of the indices of all nonnegative solutions is 1 . On the other hand, if $\left\|u_{2}\right\|_{\infty} \leqslant \gamma$, every nonnegative solution must have $v=0$, while if $\left\|u_{1}\right\|_{\infty}>\gamma$, it is unclear what the best result is. In this case, there is a $d_{1}$ in $\left(0, d_{2}\right)$ such that the equation

$$
-d h^{\prime \prime}=m\left(u_{1}-\gamma\right) h
$$

has a nontrivial positive solution satisfying the boundary conditions. Our earlier arguments imply the existence of the strictly positive solution $\left(u^{*}, v^{*}\right)$ of our equations if $d_{1}<d<d_{2}$ but the proof does not work if $0<d \leqslant d_{1}$. (The difference occurs because $\left(u_{1}, 0\right)$ has index -1 if $d>d_{1}$ but has index 0 if $d<d_{1}$.) In fact, we will see in the next section, that, in some such cases, no solution $\left(u^{*}, v^{*}\right)$ need exist for small $d$. This implies that in this case the branch of strictly positive solutions which bifurcates at $d=d_{2}$ must terminate at $d=d_{1}$ by meeting $\left(u_{1}, 0\right)$. We do not understand exactly when this behaviour occurs. Note that it is unclear in which direction the branch of solutions bifurcates at $d=d_{1}$ and, in this case, if strictly positive solutions exist for $d<d_{1}$, we would not in general expect them to be unique. We will discuss the problem further at the end of $\S 3$. Note that the difficulty in extending these results to the case where $n>1$ is in understanding the solutions of (3).

Our methods can also be used to study the existence of strictly positive solutions of other equations. For example, consider the system of equations

$$
-\Delta u=u(g-a u-b v), \quad-\Delta v=v(e-c u-d v)
$$

in $\Omega$ with Dirichlet boundary conditions. Here $a, b, c, d, e, g>0$. Our methods can be used to prove the existence of a solution with both components positive under suitable assumptions. We sketch this very briefly. For simplicity assume that $e<g$. We can obtain apriori bounds on positive solutions much as before and, also much as before, we find a unique nontrivial nonnegative solution $(\bar{u}, 0)$ which exists if $\lambda_{1}<g$. Similarly there is a unique nontrivial nonnegative solution of the form $(0, \bar{v})$ for $\lambda_{1}<e$. As before, the solution $(0,0)$ has index 0 for $\lambda_{1}<e$ and the sum of the indices of solutions is 1 (where everything is calculated with respect to $C$.) Thus, if we can prove that $(\bar{u}, 0)$ and $(0, \bar{v})$ each of index zero, it will follow that there is another solution, as required. Note that, as before, such a solution can only exist if $\lambda_{1}<$ e. Assume that

$$
A_{1}=(-\Delta+p I)^{-1}(e+p-c \bar{u}) I
$$

and

$$
A_{2}=(-\Delta+p I)^{-1}(g+p-b \bar{v}) I
$$

each have spectral radius greater than 1. (Here $p$ is a suitably large constant. As before, the conditions on the spectral radius are independent of $p$.) Note that these conditions hold if $e$ and $g$ are large while $b$ and $c$ are small. Then, as before, $(\bar{u}, 0)$ 
and $(0, \bar{v})$ each have index zero and hence there must be another solution $\left(u^{*}, v^{*}\right)$. Once again, it appears likely that this strictly positive solution $\left(u^{*}, v^{*}\right)$ is unique and stable (for the natural corresponding parabolic equations). Note also that Lazer, in some unpublished work, gives a different proof of the above result by using Schauder's fixed point theorem. However, the proof above is valid for more general classes of nonlinearities. (We only need assumptions near $u=0$, near $v=0$ and some condition giving a suitable a priori bound.)

Note that $u(\bar{x}) \leqslant a^{-1} g$ and $\bar{v}(x) \leqslant d^{-1} e$ on $\Omega$. (Similar results were proved at the beginning of §1.) It follows easily that $r\left(A_{1}\right)>1$ and $r\left(A_{2}\right)>1$ if $e-c a^{-1} g>\lambda_{1}$ and $g-b d^{-1} e>\lambda_{1}$ (since $e-c \bar{u}(x)>\lambda_{1}$ and $g-b \bar{v}(x)>\lambda_{1}$ on $\Omega$ ). Thus we see that the above result establishes the existence of a strictly positive solution under weaker assumptions than those in Leung [19] and Pao [20]. Note, however, that they discuss a number of other questions. In addition, in [19], Leung discusses more general nonlinearities. For more general nonlinearities, our results on the existence of a strictly positive solution do not include his (and vice versa). It can also be proved that there is a third nontrivial nonnegative solution if $r\left(A_{1}\right)<1$ and $r\left(A_{2}\right)<1$. (This case occurs if $e>\lambda_{1}, g>\lambda_{1}$ and $a$ and $d$ are small.) In this case, if there is only one strictly positive solution, it will not be an attractor. We do not know exactly when the system has a strictly positive solution. It is easy to see that, in order for our system to have a strictly positive solution, $g-(a x+b y)-e+(c x+d y)$ vanishes identically or changes sign on $\left\{(x, y): 0<x<\|\bar{u}\|_{\infty}, 0<y<\|\bar{v}\|_{\infty}\right\}$. Note that this problem was discussed in Brown [5] for Neumann boundary conditions.

Our methods can be applied to systems of more than 2 equations. For example, consider

$$
\begin{aligned}
-\Delta u & =u\left(a(1-u)-v_{1}-v_{2}\right), \\
-d_{1} \Delta v_{1} & =v_{1}\left(-v_{1}+m_{1}\left(u-\gamma_{1}\right)\right)+f_{1}\left(v_{1}, v_{2}\right), \\
-d_{2} \Delta v_{2} & =v_{2}\left(-v_{2}+m_{2}\left(u-\gamma_{2}\right)\right)+f_{2}\left(v_{1}, v_{2}\right)
\end{aligned}
$$

on $\Omega$ with Dirichlet boundary conditions. Here $f_{1}\left(v_{1}, v_{2}\right)>0$ if $v_{2}>0$ and $v_{1} \geqslant 0$, $f_{1}\left(v_{1}, v_{2}\right) \leqslant k_{1} v_{2}$ if $v_{1}, v_{2} \geqslant 0, f_{2}\left(v_{1}, v_{2}\right)>0$ if $v_{1}>0$ and $v_{2} \geqslant 0$ and $f_{2}\left(v_{1}, v_{2}\right) \leqslant$ $k_{2} v_{1}$ if $v_{1}, v_{2} \geqslant 0$. For simplicity, assume that $f_{1}$ and $f_{2}$ have zero derivative at $(0,0)$. Lastly assume that $k_{1} k_{2} \leqslant\left(\lambda_{1} d_{1}+m_{1} \gamma_{1}\right)\left(\lambda_{2} d_{2}+m_{2} \gamma_{2}\right)$. (The last condition implies that there are no nontrivial, nonnegative solutions with $u \equiv 0$.) Our assumptions also ensure that, if $\left(u, v_{1}, v_{2}\right)$ is a nonnegative solution with $v_{1} \equiv 0$, then $v_{2} \equiv 0$ (and vice versa). Our earlier arguments imply that there is a solution with all 3 components strictly positive if

$$
\left(-\Delta+m_{i} d_{i}^{-1} \gamma_{i} I\right)^{-1} m_{i} d_{i}^{-1} u_{a} I
$$

has spectral radius greater than 1 for $i=1$ or 2 . (The proof is simpler if we also assume that neither of the operators has spectral radius 1.) Note that the above result covers cases in which the bifurcation point, where solutions with all components positive branch off from $\left(u_{a}, 0,0\right)$, is at a double eigenvalue. Hence the local bifurcation argument in [6] is not valid at this point. 
Lastly, the invertibility requirements required to apply the index theorem in [8] are often difficult to verify. Thus we state, without proof, two simple results showing how the invertibility conditions can be weakened. Assume that $C_{i}$ is a cone in a Banach space $E_{i}, i=1,2$. Let $E=E_{1} \oplus E_{2}$ and $C=C_{1} \oplus C_{2}$. Define $C_{2, \delta}=\{v \in$ $\left.C_{2}:\|v\|<\delta\right\}$.

Proposition 2. Assume that $A: C \rightarrow C$ is completely continuous and continuously differentiable and $U \subseteq C_{1} \oplus\{0\}$ is relatively open and bounded such that $u \neq P_{1} A(u, 0)$ for $u \in \partial U$, where $P_{1}$ is the natural projection on the first factor and the boundary is relative to $C_{1}$. In addition, assume that $A(U \times\{0\}) \subseteq C_{1} \times\{0\}$. Let $T=\{u \in U$ : $\left.u=P_{1} A(u, 0)\right\}$ and $P_{2}=I-P_{1}$.

(i) Suppose that $r\left(\left.P_{2} A^{\prime}(u, 0)\right|_{R\left(P_{2}\right)}\right)>1$ for $u \in T$, that 1 is not an eigenvalue of $\left.P_{2} A^{\prime}(u, 0)\right|_{R\left(P_{2}\right)}$ corresponding to an eigenvector in $C_{2}$ for each $u \in T$ and that there is a $\delta>0$ and a number $\mu$ such that $\left.P_{2} A^{\prime}(u, v)\right|_{R\left(P_{2}\right)} \geqslant-\mu I$ (in the positive operator sense) for $u$ near $T$ and $v \in K_{2, \delta}$. Then, $\operatorname{deg}_{C}\left(I-A, U \times C_{2, \varepsilon}\right)$ is defined and equals 0 for sufficiently small positive $\varepsilon$.

(ii) If $r\left(\left.P_{2} A^{\prime}(u, 0)\right|_{R\left(P_{2}\right)}\right)<1$ for $u \in T$, then $\operatorname{deg}_{C}\left(I-A, U \times C_{2, \varepsilon}\right)$ is defined for small positive $\varepsilon$ and equals $\operatorname{deg}_{C_{1}}\left(\left.\left(I-P_{1} A\right)\right|_{R\left(P_{1}\right)}, U\right)$.

We do not give the proof here. We intend to discuss it and generalizations elsewhere. The result could be used to obtain the existence of strictly positive solutions of (2) in some cases where $n>1$ and where $f(y)=a y(b-y)(1-y)$.

Finally, assume the notation and basic conditions of Proposition 2. Suppose that $\left(u_{n}, v_{n}\right)=A\left(u_{n}, v_{n}\right)$ where $\left(u_{n}, v_{n}\right) \in C$ and $v_{n} \neq 0$ and that $\left(u_{n}, v_{n}\right) \rightarrow(\tilde{u}, 0)$ as $n \rightarrow \infty$. Then it is not difficult to show that $\left.P_{2} A^{\prime}(\tilde{u}, 0)\right|_{R\left(P_{2}\right)}$ has 1 as an eigenvalue with corresponding eigenvector in $C_{2}$. This provides a necessary condition for solutions bifurcating from the subspace $E_{1} \times\{0\}$ into $C \backslash\left(E_{1} \times\{0\}\right)$.

3. An asymptotic result. In this section, we consider the asymptotic behaviour of strictly positive solutions $\left(u^{*}, v^{*}\right)$ as $d \rightarrow 0$. By the results in $\S 1$, at least one such solution exists if $d$ is sufficiently small and $a>\lambda_{1}$. Our results prove another conjecture in [6].

As in [6], let $h(y)=f(y)-m y(y-\gamma)^{+}$. As before, $f(y)=a y(1-y)$. Note that $h$ is locally Lipschitz and $y^{-1} h(y)$ is decreasing. It turns out that the equation

$$
-\Delta u=h(u)
$$

in $\Omega$ with Dirichlet boundary conditions has no nontrivial nonnegative solution if $a \leqslant \lambda_{1}$ but has a unique nontrivial nonnegative solution $\bar{u}$ if $a>\lambda_{1}$. The nonexistence follows easily from the ideas in $\S 1$ while the existence follows easily from Theorem 2 in Dancer [7] or by the method of sub- and super-solutions. To prove uniqueness, we note that $z(x) \equiv 1$ is a supersolution and every solution must be less than or equal to $z$. Thus, by a standard argument, one can obtain a maximal solution $w$ by using the iteration $u_{n}=(-\Delta+p I)^{-1}\left(h\left(u_{n-1}\right)+p u_{n-1}\right)$ where $u_{1}=z$ and $p$ is chosen such that $h(y)+p y$ is increasing on $[0,1]$. If there is a second nontrivial nonnegative solution $v$, a simple calculation shows that

$$
-\Delta(w-v)=h(w)-h(v)<v^{-1} h(v)(w-v)
$$


in $\Omega$ since $y^{-1} h(y)$ is decreasing. (Note that $w-v$ must be positive in $\Omega$ since it is nonnegative and solves a linear elliptic equation.) As in $\S 1$, it follows that

$$
r\left((-\Delta+p I)^{-1}(p+\tilde{a}) I\right)>1, \text { where } \tilde{a}(x)=(v(x))^{-1} h(v(x)) \text { on } \Omega .
$$

However, since $-\Delta v=\left(v^{-1} h(v)\right) v$ and $v(x) \geqslant 0$ in $\Omega$, the Krein-Rutman theorem implies that $r\left((-\Delta+p I)^{-1}(p+\tilde{a}) I\right)=1$. Hence we have a contradiction and the result follows.

Theorem 2. Assume $a>\lambda_{1}$. Suppose that $d_{n} \rightarrow 0$ as $n \rightarrow \infty$ and $\left(u_{n}^{*}, v_{n}^{*}\right)$ are strictly positive solutions of (2) for $d=d_{n}$. Then $\left(u_{n}^{*}, v_{n}^{*}\right) \rightarrow\left(\bar{u}, m(\bar{u}-\gamma)^{+}\right)$in $C^{1}(\bar{\Omega})$ $\times L^{p}(\Omega)$ as $n \rightarrow \infty$ for each $p<\infty$.

REMARK. This answers another conjecture in [6]. If $\Omega$ is not smooth, $C^{1}(\bar{\Omega})$ should be replaced by $L^{\infty}(\Omega)$. We will indicate how the result can be improved after the proof.

Proof. By $\S 1,0 \leqslant u_{n}^{*}(x) \leqslant 1$ and $0 \leqslant v_{n}^{*}(x) \leqslant m(1-\gamma)$ in $\Omega$. Thus, by the first equation, $\Delta u_{n}^{*}$ is bounded in $\Omega$. By a standard regularity result (cf. Friedman [9, $\S 19])$, it follows that $u_{n}^{*}$ is bounded in $W^{2, p}(\Omega)$. Hence by the Sobolev embedding theorem (cf. Adams [1, Theorem 5.4]), $\left\{u_{n}^{*}\right\}_{n=1}^{\infty}$ is compact in $C^{1}(\bar{\Omega})$. Thus, by choosing a subsequence, we may assume that $u_{n}^{*}$ converges to $\tilde{u}$ weakly in $W^{2, p}(\Omega)$ and strongly in $C^{1}(\bar{\Omega})$. Moreover, since $\left\{v_{n}^{*}\right\}_{n=1}^{\infty}$ is bounded in $L^{p}(\Omega)$, we can choose a subsequence such that $v_{n}^{*}$ converges weakly to $\tilde{v}$ in $L^{p}(\Omega)$. Similarly, we can assume that $\left(v_{n}^{*}\right)^{2}$ converges weakly to $\tilde{w}$ in $L^{p}(\Omega)$. Since $\left\{v \in L^{\infty}(\Omega): 0 \leqslant v \leqslant\right.$ $m(1-\gamma)\}$ is strongly closed in $L^{p}(\Omega)$ and convex, it is weakly closed. Thus $\tilde{v}$ is nonnegative and in $L^{\infty}(\Omega)$. A similar property holds for $\tilde{w}$. Now

$$
\left(v_{n}^{*}-\tilde{v}\right)^{2}=\left(v_{n}^{*}\right)^{2}-2 v_{n}^{*} \tilde{v}+(\tilde{v})^{2} \rightarrow \tilde{w}-(\tilde{v})^{2}
$$

weakly in $L^{p}(\Omega)$ as $n \rightarrow \infty$. As above, it follows that $\tilde{w}-(\tilde{v})^{2} \geqslant 0$ in $\Omega$, that is, $\tilde{w} \geqslant(\tilde{v})^{2}$. The main part of our proof will consist in proving that $\tilde{w}=(\tilde{v})^{2}$.

From the first equation,

$$
-\left(u_{n}^{*}, \Delta \phi\right)=\left(a u_{n}^{*}\left(1-u_{n}^{*}\right)-u_{n}^{*} v_{n}^{*}, \phi\right)
$$

for all smooth $\phi$ of compact support in $\Omega$. Here (, ) denotes the usual scalar product. Passing to the limit, we see that

$$
(-\tilde{u}, \Delta \phi)=(a \tilde{u}(1-\tilde{u})-\tilde{u} \tilde{v}, \phi) .
$$

(For the term $\left(u_{n}^{*}, v_{n}^{*}, \phi\right)$, note that it equals $\left(v_{n}^{*}, u_{n}^{*} \phi\right)$ and note that $u_{n}^{*} \phi \rightarrow \tilde{u} \phi$ in $L^{\infty}(\Omega)$.) Thus we see that

$$
-\Delta \tilde{u}=a \tilde{u}(1-\tilde{u})-\tilde{u} \tilde{v}
$$

in $\Omega$ and $\tilde{u}(x)=0$ on $\partial \Omega$. Now the second equation becomes

$$
-d_{n}\left(v_{n}^{*}, \Delta \phi\right)=\left(-\left(v_{n}^{*}\right)^{2}+m\left(u_{n}^{*}-\gamma\right) v_{n}, \phi\right)
$$

for each smooth $\phi$ of compact support in $\Omega$. Since $v_{n}^{*}$ is bounded in $L^{\infty}(\Omega)$, the left-hand side tends to zero as $n \rightarrow \infty$ and thus we find that

$$
0=(-\tilde{u}+m(\tilde{u}-\gamma) \tilde{v}, \phi)
$$


for all smooth $\phi$ of compact support in $\Omega$. Hence $\tilde{w}=m(\tilde{u}-\gamma) \tilde{v}$. Since $\tilde{w} \geqslant(\tilde{v})^{2}$ and $\tilde{v}$ is nonnegative, it follows that $\tilde{v}(x) \leqslant m(\tilde{u}(x)-\gamma)$ when $\tilde{u}(x)>\gamma$, and $\tilde{w}(x)=\tilde{v}(x)=0$ when $\tilde{u}(x) \leqslant \gamma$.

If we can prove that $\tilde{v}(x)=m(\tilde{u}(x)-\gamma)$ when $\tilde{u}(x)>\gamma$, (7) implies that $\tilde{u}$ is a solution of (5) and thus $\tilde{u}=\bar{u}$. (Note that $\tilde{u} \neq 0$. Otherwise $-v_{n}^{*}+m\left(u_{n}^{*}-\gamma\right) \leqslant 0$ on $\Omega$ for large $n$ and the second equation gives a contradiction.) Moreover, since $\tilde{w}=m(u-\gamma) \tilde{v}$, it follows that $\tilde{w}=(\tilde{v})^{2}$. Hence by $(6),\left(v_{n}^{*}-\tilde{v}\right)^{2} \rightarrow 0$ weakly in $L^{p}(\Omega)$. Choose a test function $\phi(x)=1$ in $\Omega$. It follows that $v_{n}^{*} \rightarrow \tilde{v}$ strongly in $L^{2}(\Omega)$. If $v_{n}^{*} \rightarrow \tilde{v}$ strongly in $L^{2}(\Omega)$ and $v_{n}^{*} \rightarrow \tilde{v}$ weakly in $L^{p}(\Omega)$, Hölder's inequality implies that $v_{n}^{*} \rightarrow \tilde{v}$ strongly in $L^{q}(\Omega)$ for $2<q<p$. Thus the result reduces to proving that $\tilde{v}(x) \geqslant m(\tilde{u}(x)-\gamma)$ a.e. where $\tilde{u}(x)>\gamma$. (Note that the whole sequence must converge because every subsequence has the same limit.)

To prove this, we use subsolutions again. Suppose $\varepsilon>0$. Choose $\mu>0$ such that $|\tilde{u}(x)-\tilde{u}(y)| \leqslant \frac{1}{3} \varepsilon m^{-1}$ if $x, y \in \bar{\Omega}$ and $\|x-y\| \leqslant \mu$. Hence, if $n$ is large, $\mid u_{n}^{*}(x)-$ $u_{n}^{*}(y) \mid \leqslant \frac{1}{2} \varepsilon m^{-1}$ for $\|x-y\| \leqslant \mu$. Suppose $x_{0} \in \Omega$ and $\tilde{u}(x)>\gamma$ if $\left\|x-x_{0}\right\| \leqslant \mu$. Let $\alpha=m\left(\tilde{u}\left(x_{0}\right)-\gamma\right)-\varepsilon$, let $B$ denote the ball of radius $\mu$ with centre $x_{0}$, and let $\tilde{B}$ be the concentric ball of half the radius. Choose a smooth function $h$ such that $h(x)=0$ on $\partial B, 0<h(x) \leqslant \alpha$ inside $B, h(x)=\alpha$ on $\tilde{B}$, and $|\Delta h| \leqslant K h$ in $B$. Let $w(x)=h(x)$ in $B$ and 0 otherwise. By a tedious calculation we find as in $\S 1$ (cf. [13]) that $w$ is a subsolution of

$$
-\Delta v=d_{n}^{-1} v\left(-v+m\left(u_{n}^{*}-\gamma\right)\right)
$$

if $n$ is large. (Note that $d_{n}$ is small and $m\left(u_{n}^{*}(x)-\gamma\right) \geqslant \alpha+\frac{1}{2} \varepsilon$ on B.) Thus (cf. Lemma 1 of $\S 1$, the unique solution $\tilde{v}_{n}$ of (8) satisfies $\tilde{v}_{n} \geqslant w$. However, this unique solution is $v_{n}^{*}$. Thus $v_{n}^{*} \geqslant w$ for large $n$. Hence $\tilde{v} \geqslant w$ a.e. on $B$. In particular, $\tilde{v} \geqslant \alpha$ a.e. on $\tilde{B}$. In other words $\tilde{v}(x) \geqslant m\left(\tilde{u}\left(x_{0}\right)-\gamma\right)-\varepsilon$ a.e. on $\tilde{B}$. Hence

$$
\tilde{v}(x) \geqslant m(\tilde{u}(x)-\gamma)-2 \varepsilon \quad \text { a.e. on } \tilde{B}
$$

(by the definition of $\mu$ ). Thus we see that $\tilde{v}(x) \geqslant m(\tilde{u}(x)-\gamma)-2 \varepsilon$ a.e. where $d(x, T) \geqslant \mu$. Here $T=\{x \in \Omega: \tilde{u}(x) \leqslant \gamma\}$. Since we can choose $\mu$ small if $\varepsilon$ is small, it follows that $\tilde{v}(x) \geqslant m(\tilde{u}(x)-\gamma)$ a.e. on $\Omega \backslash T$. This completes the proof.

Theorem 2 can be improved. By considering where the function $v_{n}^{*}(x)-$ $m\left(u_{n}^{*}(x)-\gamma\right)$ has its minimum or by examining carefully the last part of the proof, we see without difficulty that, if $\varepsilon>0$, then $v_{n}^{*}(x)-m\left(u_{n}^{*}(x)-\gamma\right) \geqslant-\varepsilon$ on $\Omega$ for $n$ large. Conversely, let $w(x)=\sup \{\varepsilon, m(\bar{u}(x)-\gamma)+\varepsilon\}$. Then $w$ is $C^{2}$ except where $\bar{u}(x)=\gamma$. By perturbing $w$ by at most $\frac{1}{4} \varepsilon$ near $\{x: \bar{u}(x)=\gamma\}$, we can obtain a $C^{2}$ function $\bar{w}$ such that $\bar{w}(x)(-\bar{w}(x)+m(\bar{u}(x)-\gamma)) \leqslant-\frac{1}{2} \varepsilon^{2}$ on $\Omega$. Thus, for $n$ large, we see by a simple calculation that $\bar{w}$ is a supersolution of the equation satisfied by $v_{n}^{*}$. Hence $v_{n}^{*}(x) \leqslant \bar{w}(x)$ on $\Omega$ for $n$ large. This and the first result of the paragraph imply that $v_{n}^{*} \rightarrow m(\bar{u}(x)-\gamma)^{+}$in $C(\bar{\Omega})$ as $n \rightarrow \infty$. Hence there is no boundary layer.

Note that the proof of Theorem 2 is quite insensitive to the dependence upon $u$ in the first equation. In particular, it applies if we take $f(y)$ to be $a y(y-b)(1-y)$ instead of $a y(1-y)$. One slight difference occurs. If (5) has more than one solution $u$ with $\|u\|_{\infty} \geqslant \gamma$, then the sequence $\left\{\left(u_{n}^{*}, v_{n}^{*}\right)\right\}_{n=1}^{\infty}$ has

$$
\left\{\left(\tilde{u}, m(\tilde{u}-\gamma)^{+}\right): \tilde{u} \text { is a solution of }(5),\|\tilde{u}\|_{\infty} \geqslant \gamma\right\}
$$


as its possible limit points. If (5) has no nontrivial solution $u$ with $\|u\|_{\infty} \geqslant \gamma$, it follows that (2) has no solutions with both components positive for $d$ sufficiently small. This was mentioned in $\S 2$. Note that (5) has no nontrivial nonnegative solutions if $h(y)<\lambda_{1} y$ on $(0,1)$. (For example, this holds if $\gamma$ is near $b$ and $m$ is large.) In the case where $n=1$, this property could be discussed in more detail by the methods of [6]. (Some discussion of this property appears in [6].)

It is possible to show that, if $w$ is an isolated nonnegative solution of (5) of nonzero index $k$ and if $\|w\|_{\infty}>\gamma$, then there is a solution $\left(u^{*}, v^{*}\right)$ near $\left(w, m(w-\gamma)^{+}\right)$for all sufficiently small positive $d$. Moreover, the sum of the indices of such solutions is $k$. (This is proved by using Theorem 2 and degree theory and by deforming (2) to a system where $v$ in the first equation is replaced by $m(u-\gamma)^{+}$and $u$ in the second equation is replaced by $w$. Once again, this proof is quite insensitive to the formula for $f$.

The above result can be used to construct examples where $n=1, \Omega=(-L, L)$, $f(y)=a(1-y)(y-b)$ and there is relatively complicated solution structure. We consider cases where (3) has 2 distinct nonnegative solutions $u_{1}, u_{2}$ with $\left\|u_{1}\right\|_{\infty}>\gamma$ and $u_{2}>u_{1}$ and where (5) has 2 distinct nonnegative nontrivial solutions $\tilde{u}_{1}, \tilde{u}_{2}$.We assume these conditions hold for the remainder of the paragraph. As before, we consider $d$ as a parameter. There are examples where the solution branch $B_{1}$ of strictly positive solutions branching from $\left(u_{1}, 0\right)$ at $d=d_{1}$ continues to exist for all $d$ in $\left(0, d_{1}\right)$. Similar properties hold for the branch $B_{2}$ bifurcating from $\left(u_{2}, 0\right)$ at $d=d_{2}$. Moreover, these branches do not intersect and have distinct limits as $d \rightarrow 0$. (Note that $d_{1}$ and $d_{2}$ were defined earlier in \$2.) In particular, there are two distinct strictly positive solutions for all $d$ in $\left(0, d_{1}\right)$. (To construct such an example, one shows that, if $m$ is small, then, for any strictly positive solution $(u, v)$ of $(2), u$ is near $u_{1}$ or $u_{2}$. Moreover, this holds uniformly in $d$.) Secondly, there are examples where there are $d$ 's in $\left(0, d_{1}\right)$ for which (2) has no strictly positive solution. It follows in this case that the branch $B_{1}$ of strictly positive solutions bifurcating from $\left(u_{1}, 0\right)$ at $d=d_{1}$ has $\left(u_{2}, 0\right)$ in its closure and $\inf \left\{d:(u, v, d) \in B_{1}\right\}>0$. Moreover, the branch of solutions $\tilde{B}_{1}$ bifurcating from $\left(\tilde{u}_{1}, 0\right)$ at $d=0$ must return to $\left(\tilde{u}_{2}, 0\right)$ (for $d=0$ ) and $\tilde{B}_{1} \cap B_{1}=\varnothing$. (To construct such an example one starts with $a$ such that $u_{1}=u_{2}$, increases $a$ slightly and chooses $m$ very small. One also uses the result that, if (3) has a unique nontrivial nonnegative solution, then every nonnegative solution of (2) has $v=0$. This last result is proved by combining some of the ideas in $\S 1$ with those in [8].) Finally, other more complicated solution structures must occur. If we deform the parameters $a, m, \gamma$ for the first example in the paragraph to those for the second in such a way that the basic assumptions of this paragraph hold throughout the deformation, there must be a place where the solution structure changes. (Note that it can be proved that such a deformation exists.)

\section{REFERENCES}

1. R. A. Adams, Sobolev spaces, Academic Press, New York, 1975.

2. H. Amann, Fixed point equations and nonlinear eigenvalue problems in ordered Banach spaces, SIAM Rev. 18 (1976), 620-709.

3. N. Anselone, Collectively compact operator approximation theory and applications to integral equations, Prentice-Hall, Englewood Cliffs, N. J., 1971. 
4. H. Berestycki, Le nombre de solutions de certains problèmes semilinéaires elliptiques, J. Funct. Anal. 40 (1981), 1-29.

5. P. N. Brown, Decay to uniform states in ecological interactions, SIAM J. Appl. Math. 38 (1980), 22-37.

6. E. Conway, R. Gardner and J. Smoller, Stability and bifurcation of steady state solutions for predator-prey equations, Adv. in Appl. Math. 3 (1982), 288-334.

7. E. N. Dancer, Global solution branches for positive mappings, Arch. Rational Mech. Anal. 52 (1973), $181-192$.

8. $\ldots$ On the indices of fixed points of mappings in cones and applications, J. Math. Anal. Appl. 91 (1983), 131-151.

9. A. Friedman, Partial differential equations, Holt, Rinehart and Winston, New York, 1969.

10. P. Hess and T. Kato, On some linear and nonlinear eigenvalue problems with an indefinite weight function, Comm. Partial Differential Equations 10 (1980), 999-1030.

11. T. Kato, Perturbation theory for linear operators, Springer-Verlag, Berlin, 1966.

12. M. A. Krasnosel'skii, Positive solutions of operator equations, Noordhoff, Groningen, 1964.

13. P. L. Lions, On the existence of positive solutions of semilinear elliptic equations, SIAM Rev. 24 (1982), 441-448.

14. G. Pimbley, Eigenfunction branches of nonlinear operators and their bifurcations, Lecture Notes in Math., vol. 104, Springer-Verlag, Berlin, 1969.

15. P. H. Rabinowitz, Some global results for nonlinear eigenvalue problems, J. Funct. Anal. 7 (1971), 487-513.

16. W. Rudin, Real and complex unalysis, McGraw-Hill, New York, 1970.

17. H. H. Schaefer, Topological vector spaces, Macmillan, New York, 1966.

18. J. Smoller and H. Wasserman, Global bifurcation of steady state solutions, J. Differential Equations 39 (1981), 269-290.

19. A. Leung, Equilibria and stabilities for competing species reaction-diffusion equations with Dirichlet boundary data, J. Math. Anal. Appl. 73 (1980), 204-218.

20. C. V. Pao, Coexistence and stability of a competition diffusion system in population dynamics, J. Math. Anal. Appl. 83 (1981), 54-76.

Department of Mathematics, University of New England, Armidale, New South Wales 2351, Australia 